\title{
ATTRACTORS WITH POSITIVELY LYAPUNOV STABLE TRAJECTORIES
}

\author{
TADEUSZ NADZIEJA
}

\begin{abstract}
We show that a finite dimensional attractor in locally connected metric space consisting of positively Lyapunov stable trajectories is a torus.
\end{abstract}

There appear in physics, chemistry and biology deterministic systems $\dot{x}=f(x)$ with an irregular, chaotic, turbulent time evolution. This phenomenon has received various mathematical interpretations $[5,6,10,9,11]$.

According to the theory of Landau [6] the turbulent motion is asymptotically given by a quasi periodic function of time $x(t)=f\left(\omega_{1} t, \ldots, \omega_{n} t\right)$, where $f$ has period 1 in each argument separately and the frequences $\omega_{1}, \ldots, \omega_{n}$ are not rationally related. The attractors in such systems are tori.

Ruelle and Takens [11] argue that the occurrence of a strange attractor provides a mechanism for understanding turbulence. No definition of strange attractor is universally accepted. In [11] it is an attracting set which is compact, invariant and connected but is not a point, circle or a surface of any dimension. Ruelle $[9,10]$ assumes that on a strange attractor all trajectories have sensitive dependence on initial data i.e. trajectories are Lyapunov unstable.

In the investigation of systems with complex behavior, in particular turbulent flows, it seems reasonable to assume that the process takes place in some metric space and all the limit sets of trajectories lie in a single compact set. If a strange attractor occurs in a system, then the system is turbulent in the Ruelle-Takens sense.

A natural question arises: What attractors may occur in systems without RuelleTakens turbulence? All the attractors in such systems consist of positively Lyapunov stable points. We will characterize these attractors in this note.

Let $(X, d)$ be a metric space. By $\operatorname{cl} A$ we denote the closure of a set $A \subset X, K_{r}(p)$ is the open ball of radius $r$ centered at $p$. A flow on $X$ is a continuous map $f: R \times X \rightarrow X$ ( $R$ real numbers), satisfying the conditions: $f(0, x)=x$ for $x \in X$ and $f(s+t, x)=f(t, f(s, x))$ for $x \in X$ and $t, s \in R$. The orbit of a point $p \in X$ is the set $T(p)=\{f(t, p): t \in R\}$ and the half positive trajectory is the set $T^{+}(p)=$ $\{f(t, p): t \geqslant 0\}$. For any $p \in X$ the set $\omega(\alpha)(p)=\left\{q \in X\right.$ : there is a sequence $\left\{t_{n}\right\}$ with $t_{n} \rightarrow+(-) \infty$ and $\left.\lim _{n \rightarrow+\infty} f\left(t_{n}, p\right)=q\right\}$ is called its positive (negative) limit set [2]. A subset $A \subset X$ is invariant if $f(t, A) \subset A$ for all $t \in R$.

Received by the editors March 31, 1981.

1980 Mathematics Subject Classification. Primary 34D20, 58F12.

Key words and phrases. Attractor, turbulence, positively Lyapunov stable trajectory, almost periodic orbit. 
We shall say that an invariant, compact subset $A \subset X$ is an attractor if:

a. for every neighbourhood $U$ of $A$, there is a neighbourhood $V$ of $A$ such that $f(t, V) \subset U$ for all positive $t$ and $\omega(x) \subset A$ for every $x \in V$,

b. there exists a point $p \in A$ such that $\operatorname{cl} T(p)=A$.

The domain of attraction $D(A)$ of an attractor $A$ is the set of all points $x \in X$ such that $\omega(x) \subset A$. It is easy to see [2] that $D(A)$ is open.

A point $q \in X$ is positively Lyapunov stable [7] (l-stable) if for every $\varepsilon>0$ there is $\delta>0$ such that if $d(q ; x)<\delta$ then $d(f(t, q), f(t, x))<\varepsilon$ for $t \geqslant 0$. If a point $q \in X$ is $l$-stable then the point $f(t, q)$ is $l$-stable for every $t \in R$. Hence it is meaningful to say that the orbit of the point $q \in X$ is positively Lyapunov stable.

For the remainder of this paper $\mathcal{L}$ will denote an attractor all of whose points are l-stable.

LEMMA 1. There exists a point $p \in \mathcal{L}$ such that $\operatorname{cl} T^{+}(p)=\mathcal{L}$.

Proof. By assumption, there exists a point $p \in \mathcal{L}$ such that $\operatorname{cl} T(p)=\mathcal{L}$. We will show that if $q \in \alpha(p)$ then $\omega(q) \supset T(p)$. Let $x=f\left(t_{1}, p\right)$ and pick $\varepsilon>0$. Since $q \in \alpha(p)$ and $p$ is $l$-stable, for every real $K$ there exists $t_{2}<K$ and $t_{2}<t_{1}$ such that $d\left(f\left(t_{2}+t, p\right), f(t, q)\right)<\varepsilon$ for $t \geqslant 0$. Hence $d\left(x, f\left(t_{1}-t_{2}, q\right)\right)<\varepsilon$, which proves our inclusion. $\alpha(p)$ being invariant and closed we thus have $\alpha(p) \supset \omega(p)$ and so $\omega(p) \supset T(p)$. This together with the inclusion $\operatorname{cl} T^{+}(p) \supset \omega(p)$ implies $\operatorname{cl} T^{+}(p)$ $=\mathfrak{e}$.

A compact, invariant set $A$ is called minimal if it contains no proper invariant, closed subset. It is easy to show [2] that a compact set $A$ is minimal if and only if $\operatorname{cl} T(p)=A$ for every point $p \in A$.

LEMMA 2. $\mathcal{E}$ is a minimal set.

Proof. Pick $q \in \mathcal{L}$ and $\varepsilon>0$. By Lemma 1 there exists a point $p_{1} \in \mathcal{E}$ such that $\operatorname{cl} T^{+}\left(p_{1}\right)=\mathcal{L}$ and $d\left(f\left(t, p_{1}\right), f(t, q)\right)<\varepsilon$ for $t \geqslant 0$. Since $\varepsilon$ is arbitrary we have $\operatorname{cl} T^{+}(q)=\mathscr{e}$.

The point $p \in X$ will be called almost periodic if for every $\varepsilon>0$ there exists a relatively dense subset of numbers $\left\{\tau_{n}\right\}$ such that $d\left(f(t, p), f\left(t+\tau_{n}, p\right)\right)<\varepsilon$ for all $t \in R$ and each $\tau_{n}$.

REMARK 1. It was proved in [7, Theorem 8.04] that if $B$ is a compact set consisting of $l$-stable points then for every $\varepsilon>0$ there is a number $\delta>0$ such that $d(f(t, p), f(t, q))<\varepsilon$ for all $t \geqslant 0$ if $p \in B$ and $d(p, q)<\delta$.

REMARK 2. It follows from Lemma 2 that if $q \in D(\mathcal{L})$ then $\omega(q)=\mathfrak{L}$.

Using Theorem 5 of [3] and two last remarks we conclude that $\mathcal{L}$ is a minimal set of almost periodic points.

REMARK 3. Corollary 8.09 of [7] implies that for any $r>0$ there exists a number $\delta(r)>0$ such that for $q_{1}, q_{2} \in \mathcal{L}, d\left(q_{1}, q_{2}\right)<\delta(r)$ implies $d\left(f\left(t, q_{1}\right), f\left(t, q_{2}\right)\right)<r$ for $t \in R$.

An easy consequence of Remark 3 is:

REMARK 4. For any $q_{1}, q_{2} \in \mathcal{L}, q_{1} \neq q_{2}$ there is a number $a>0$ such that $d\left(f\left(t, q_{1}\right), f\left(t, q_{2}\right)\right)>a$ for $t \in R$. 
LEMMA 3. The set $D(\mathcal{L})$ consists of l-stable points.

Proof. From Remark 1 it follows that for every $\varepsilon>0$ there exists $\delta(\varepsilon)>0$ such that $d(f(t, p), f(t, q))<\varepsilon$ for all $t \geqslant 0$ if $p \in \mathcal{L}$ and $d(p, q)<\delta(\varepsilon)$. Let $y \in D(\mathcal{L})$ and $\varepsilon>0$. We pick a number $r>0$ such that $K_{r}(y) \subset D(\mathcal{L})$ and for some $T>0$ $\operatorname{dist}(f(t, x), \mathcal{L})<\frac{1}{2} \delta(\varepsilon / 2)$ for $x \in K_{r}(y)$ and $t>T$. The existence of such $T$ follows from definition of the attractor [2]. By continuity of flow we may choose $\tilde{r}<r$ such that $d(f(t, x), f(t, y))<\frac{1}{2} \delta(\varepsilon / 2)$ for $x \in K_{r}(y)$ and $t \in[0, T]$. It is easy to see that $d(f(t, x), f(t, y))<\varepsilon$ for all $t \geqslant 0$ and $x \in K_{r}(y)$.

For any $p \in \mathcal{L}$ we define

$$
W^{s}(p)=\left\{x \in D(\mathcal{L}): \lim _{t \rightarrow+\infty} d(f(t, x), f(t, p))=0\right\} .
$$

Remark 4 implies that if $p_{1} \neq p_{2}$ then $W^{s}\left(p_{1}\right) \cap W^{s}\left(p_{2}\right)=\varnothing$.

LEMMA 4. $D(\mathfrak{L})=\cup\left\{W^{s}(p): p \in \mathcal{E}\right\}$.

Proof. Let $x \in D(\mathcal{L})$ and $t_{n} \rightarrow+\infty$. Since $\omega(x) \subset \mathcal{L}$ we may choose a subsequence $t_{n_{k}} \rightarrow+\infty$ such that $\lim _{k \rightarrow+\infty} f\left(t_{n_{k}}, x\right)=z \in \mathcal{L}$. Consider the sequence $z_{n_{k}}=f\left(-t_{n_{k}}, z\right)$. Due to $z_{n_{k}} \in \mathcal{L}$ one can choose a convergent subsequence $z_{n_{k^{\prime}}}$. Suppose $\lim _{k^{\prime} \rightarrow+\infty} z_{n_{k^{\prime}}}=p$. From $l$-stability of the point $p$ it follows that $\lim _{k^{\prime} \rightarrow+\infty} d\left(f\left(t_{n_{k^{\prime}}}, p\right), f\left(t_{n_{k^{\prime}}}, x\right)\right)=0$. The last equality and $l$-stability of $p$ imply $x \in W^{s}(p)$.

LEMMA 5. If $p \in \mathcal{L}$, then for every neighbourhood $U$ of $p$, there exists a smaller neighbourhood $V$ such that $x \in V$ and $x \in W^{s}(q)$ imply $q \in U$.

Proof. Let $U$ be a neighbourhood of $p \in \mathcal{L}$. We may suppose that $U$ is an open ball $K_{r}(p)$ and $K_{r}(p) \subset D(\mathfrak{E})$. From $l$-stability of $p$ it follows that there is a number $\tilde{\delta}>0$ such that if $x \in K_{\tilde{\delta}}(p)$ then $d(f(t, x), f(t, p))<\frac{1}{2} \delta(r)$ for $t \geqslant 0(\delta(r)$ corresponds to $r$ as in Remark 3). Let $x \in K_{\tilde{\delta}}(p)$ and $x \in W^{s}(q)$. For sufficiently large $\bar{t}$ we have $d(f(\bar{t}, q), f(\bar{t}, x))<\frac{1}{2} \delta(r)$, hence $d(f(\bar{t}, q), f(\bar{t}, p))<\delta(r)$ which by definition of $\delta(r)$ implies $d(f(-\bar{t}, f(\bar{t}, q)), f(-\bar{t}, f(\bar{t}, p)))=d(q, p)<r$.

$\mathcal{L}$ is a minimal set of almost periodic points hence we may equip $\mathcal{L}$ with a structure of a compact topological group as in [7]. From now on we will suppose that covering dimension [4] of $E$ is finite. In [8] it was shown that a commutative connected finite dimensional compact group is locally homeomorphic to set $B=G \times n$-cell where $G$ is a compact 0 -dimensional topological group. We have two possibilities, either $G$ is a discrete or $G$ is a perfect set. Since a perfect, 0 -dimensional subset of a metric

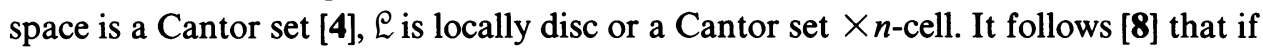
$\mathcal{L}$ is locally connected then $\mathcal{L}$ is a torus.

THEOREM 1. A finite dimensional attractor $\mathcal{L}$ contained in a locally connected metric space consisting of positively Lyapunov stable points is a torus.

Proof. We must show that $\mathcal{L}$ is locally connected. If it is not so, every point $p \in \mathcal{E}$ has a neighbourhood $U$ such that $U \cap \mathcal{E}$ is the product of $n$-cell and a Cantor set. Let $p \in \mathcal{L}$ and $V$ be a connected neighbourhood of $p$. By Lemma 5 we may suppose that $V \subset \cup\left\{W^{s}(q): q \in \mathcal{L} \cap U\right\}$. Since $U \cap \mathcal{L}$ is a product of a Cantor set and 
$n$-cell, $U \cap \mathcal{L}$ may be decomposed into a sum of two sets $\mathcal{L}_{1}, \mathcal{L}_{2}$ such that $\mathcal{L}_{i} \cap V \neq \varnothing$, $i=1,2$, and $d\left(p_{1}, p_{2}\right)>r>0$ for every $p_{1} \in \mathcal{L}_{1}$ and $p_{2} \in \mathcal{L}_{2}$. Now we put $V_{i}=$ $\cup\left\{W^{s}(q): q \in \mathcal{L}_{i}\right\} \cap V$. We will show that $V_{i}$ is open. Let $y \in W^{s}(q) \cap V$ and $q \in \mathcal{L}_{i}$. From Lemma 3 it follows that there exists $\varepsilon>0$ such that if $d(x, y)<\varepsilon$ then $d(f(t, x), f(t, y))<\frac{1}{2} \delta(r)$ for $t \geqslant 0(\delta(r)$ corresponds to $r$ as in Remark 3). If $x \in K_{\varepsilon}(y) \cap V \cap W^{s}(p)$ then $d(f(t, p), f(t, q))<\delta(r)$ for sufficiently large $t$. Hence $d(p, q)<r$. The last inequality and Lemma 5 imply $x \in V_{i}$. Of course $V_{1} \cap V_{2}=\varnothing$ and $V_{1} \cup V_{2}=V$. We have decomposed the set $V$ into the sum of two open, nonempty sets. This contradicts $V$ is connected.

REMARK 5. It is shown in [1], with the aid of the homology theory that if $X$ is a differentiable manifold then a minimal set of almost periodic points which is an attractor is a torus. Thus in this case Theorem 1 follows from this result.

It has been shown in [7] that on every compact metric group $S$ there exists a flow, such that $S$ is a minimal set of almost periodic points. Hence any such group may be an attractor consisting of $l$-stable points. Thus our assumption that $L$ is contained in a locally connected metric space cannot be relaxed.

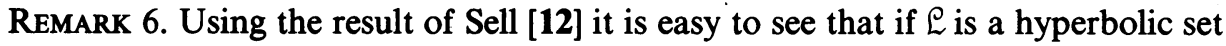
contained in a differentiable manifold $X$ then $\mathcal{E}$ is a point or periodic orbit.

ACKNowledgment. I would like to thank Professor A. Krzywicki for discussions during the preparation of this paper. I am also indebted to the referee for helpful suggestions.

\section{REFERENCES}

1. G. Allaud and E. S. Thomas, Almost periodic minimal sets, J. Differential Equations 15 (1974), $158-171$.

2. N. P. Bhatia and G. P. Szegö, Stability theory of dynamical systems, Springer-Verlag, Berlin, 1970.

3. L. G. Deysach and G. R. Sell, On the existence of almost periodic motions, Michigan Math. J. 12 (1965), 87-95.

4. R. Engelking, Topologia ogólna, PWN, Warsaw, 1975.

5. J. L. Kaplan and J. A. Yorke, The onset of chaos in a fluid flow model of Lorenz, Ann. New York Acad. Sci. 316 (1978), 400-407.

6. L. D. Landau and E. M. Lifschitz, Fluid mechanics, Pergamon Press, Oxford, 1959.

7. V. V. Nemytskii and V. V. Stepanov, Qualitative theory of differential equations, Princeton Univ. Press, Princeton, N.J., 1960.

8. L. S. Pontryagin, Topological groups, 2nd ed., Gordon and Breach, New York, 1966.

9. D. Ruelle, Sensitive dependence on initial conditions and turbulent behavior of dynamical systems, Ann. New York Acad. Sci 316 (1978), 408-416.

10. __ Strange attractors, Math. Intell. 2 (1980), 126-137.

11. D. Ruelle and F. Takens, On the nature of turbulence, Comment. Math. Phys. 20 (1971), 167-192.

12. G. R. Sell, Periodic solutions and asymptotic stability, J. Differential Equations 2 (1966), 143-157.

Institute of Mathematics, Wroclaw University, 50384 Wroclaw, Plac GrunWaldzki 2/4, POLAND 